quence of the attrition exercised upon it for a considerable time by the head of the humerus, is by no means an uncommon circumstance. Amongst collections of diseased bones, it is not rare to find porcelainous deposits on the under surface of the acromion, as well as on the articular surfaces naturally entering into the formation of the shoulder-joint. In the museum of University College, for instance, there are six specimens illustrating this fact. And to come more nearly to the point, of five old dislocations of the shoulder, examined and described by Mr. Smith, (Medical Gazette, vol. xiv.,) in which the long tendon of the biceps was ruptured, no less than four presented this appearance on the acromion. In two other cases of dislocation, described in the same paper, no such false joint existed, but in both these instances the tendon of the biceps remained entire, though displaced from its groove.

These three conditions can, then, only be considered as evidence that the head of the humerus was thrown in an upward direction, but nothing further. Let us next inquire what facts should assist us in determining whether it was originally directly upwards, or upwards and forwards.

The first circumstance tending to throw a doubt over the former of these two suppositions, is the vast amount of injury inflicted on the ligaments and tendons around the joint, without any corresponding evidence of violence having been exercised on the acromion process. For although, in Mr. Smee's case fracture of the clavicle was found on the same side, there is nothing to prove that it occurred at the time of the dislocation nor is it the precise kind of injury that would be likely to occur from a direct and rapid application of force from below, or the end of the acromion. In order to produce so much laceration, not only of the muscles but of the capsular ligament, the head of the humerus must (one would think) have passed for some considerable distance from its natural position; and how it could find room for this directly upwards, without splintering off the acromion in its passage, is a problem somewhat difficult of solution.

The facts, however, which most strongly support the view of the dislocation haviug primarily been forwards and upwards, are derived from a consideration of the state of the muscles around the joint.

The state of the muscles in the more common forms of dislocation has now been examined in many recent cases, and has been found, for the most part, to correspond so well with what migh be expected from an $\grave{a}$ prior $i$ consideration of the course and attachment of these muscles, that, knowing the direction in which the bone has been displaced, we may, with tolerable certainty, name the muscles most likely to be found torn, or put violently on the stretch.

The converse of this cannot, however, so readily be inferred; that is, knowing the muscles torn across, we cannot in every case infer, from such facts alone, in what direction the head of the bone escaped. This difficulty arises from the circumstance that (owing probably to individual differences in the size and strength of these muscles, the precise direction of the displacing force, and the state of action of particular muscles at the moment of displacement) the greatest variety is found to exist both as to the number of muscles torn and as to the extent to which they are injured.

To take an example:-In a case described by Mr. Curling, (Sir Astley Cooper on Dislocations, last edition, p. 385, ) of dislocation of the head of the humerus forwards and upwards, the supraspinatus, infra-spinatus, and sub-scapularis muscles were all torn across at their attachments; whilst in a case related by Mr. Key, (in the same work, ) and in another recorded by Sir Philip Crampton, (loc. cit.) of the same kind of dislocation, none of the muscles were ruptured, although the supra-spinatus and infraspinatus were put violently on the stretch.

Nor is this at all surprising, when we consider the great variety of circumstances under which such accidents may occur. In one instance the muscles may act so suddenly and powerfully, and the force may be applied so rapidly, that the parts tear before they can yield; whilst in another case, from the opposite conditions, the corresponding muscles, taken, as it were, by surprise, yield instead of tearing, or offer just sufficient resistance at the moment to cause the head of the bone to roll a little on one side, and pierce the capsule at an interval between the tendons.

Where muscles inserted into opposite sides of a displaced bone are both ruptured, the rupture must be brought about in two different modes; one set of muscles being pushed in front of the advancing bone, the other set being dragged after it; the one being made tense over the head of the bone displaced, the other being stretched over the cavity from which the bone is dislodged.

Of these, however, the latter appears to me by far the more likely to occur, inasmuch as the head of the bone, whilst pushing the muscle before it, and forcing it to describe a curve, tends at the same time to bring the two extremities of the muscle nearer together, and thus causes less actual tension than might at first be supposed.

If this is the case, we may infer that where rupture of fibres, or fracture of the bony attachment, is limited to one group of muscles, (the opposing group retaining their natural insertions and healthy appearance, the head of the bone has probably taken a direction away from the muscles so injured, and towards those that have escaped injury.

To apply this to the cases under consideration. It is remarkable, that in both instances the sub-scapular muscle escaped injury, although the supra and infra-spinati were completely separated from their insertions. Under the supposition that the dislocation was directly upwards, it is difficult to explain why there should have been this great difference, unless, indeed, at the time of the accident, the arm had been strongly rotated outwards. Even then I am at a loss, considering the relation of the parts, to understand why the supra-spinatus should have received so much injury, when the teres minor escaped altogether.

If, however, we suppose the displacement to have occurred upwards and forwards, would not this difierence in the amount of injury produced be more satisfactorily accounted for?

I am aware it may be said that this is only arguing on probabilities, but, unfortunately, the facts collected from recent cases are so meagre on this particular point, that it is the only mode we possess of approaching the question.

The positions I have endeavoured to maintain are-1st, That there is not sufficient evidence in the cases before us, to conclude that they were instances of the rare form of primary dislocation upwards; and, 2ndly, That there are reasons for believing that they were originally displacements of the head of the humerus upwards and forwards.

Langham-place, Regent-strect, April 7, 1845.

\section{RETENTION OF URINE PRODUCED BY A LARGE QUANTITY OF GREY PEAS IN THE RECTUM.}

By George Todn, Esq. Surreon, Durham.

SAnuer B-, aged sixty-two, of good constitution, after having partaken three times of peas-pudding, made of whole grey peas, at different times during the day on the 20th of March, 1844, was unable to pass any urine during the night and following day. On the evening of the 21 st, I was called to him, and found the patient complaining of great pain over the lower part of the abdomen, great tenesmus, and a constant desire to void his urine. The bladder was distended, and there was some tenderness on pressure being made over the pubic region. "I introdnced the catheter without any difficulty, and drew off about two pints of dark-coloured urine, which afforded great relief, but the patient was sure that all his sufferings arose from the peas in his rectum. I administered a common purgative enena, which did not pass far up the bowel, and was not retained, nor did it produce any fæcal evacuation. He took an anodyne, and was ordered castor oil early the following morning.

22nd.-Has not passed any urine during the night, and has been rnuch disturbed in consequence of constant tenesmus, and a feeling as if something was blocking up the rectum. I introduced the catheter, and administered a purgative injection, which, however, was not retained, and no evacuation of any consequence was procured. He was ordered to take castor oil, followed by a strong purgative mixture, at intervals during the day.

23rd.- Has passed an easy night, but has not passed any urine, nor had any evacuation from the bowels, with the exception of a few grey peas. I introdnced the catheter, and drew off about a pint of healthy-looking urine. During the day, I administered a great variety of purgatives, including a large dose of croton oil. Evening.-Has not passed any urine, nor had any evacuation from the bowels. Complains of much tenesmus, and there is some constitutional disturbance. I now set to work to administer strong purgative injections, and to endeavour, if possible, to dislodge the cause of obstruction. I had given him two injections without producing any action of the bowels, and was administering the third, when the rectum contracted, and with great force expelled an enormous quantity of whole grey peas. They were expelled with such force as to be sent to every part of the room, and resembled as many short corks coming from an exploded gun. I administered another enema, which brought away more peas. He passed a comfortable night, and in the morning was able to evacuate his bladder without my assistance. After this he was soon well.

Remarks. - In this case I was at a loss to understand the cause of the obstruction in the bowels, and as it was evident that this 
obstruction was the cause of the retention of urine, it became absolutely necessary that this intestinal obstruction should be overcome. The patient from the very first was convineed that all his sufferings arose from the peas, but I had no idea that such a large collection could have taken place, and I fancied that the obstruction was occasioned by a quantity of hardened fæes in the rectum.

April, 1845.

\section{BRITISH AND AMERICAN MEDICAL JOURNALS.}

THE DIAGNOSIS OF HEPATITIS AND HEPATALGIA.

The following summary, though it has nothing novel to recommend it, may be advantageously borne in mind.-

"Although the pains accompanying hepatalgia may be as intense as those of hepatitis, and in many instances, perhaps, more urgent, they are not constant, but are at the outset, and frequently also, during the whole progress of the disorder, paroxysmal, affording in the interval a complete immunity from pain. The pathognomonic signs indicative of inflammatory action of the liver, are pyrexia, tumefaction, great tenderness in the hypochondrium, frequent and strong pulse, thirst, furred tongue, and vomiting, sometimes of a bilions, and at other times of a dark-coloured, secretion, as the substance of the liver more or less partakes of the invading disease. The bowels are irregular in their action, the evacuations presenting a great variety of appearances, according as the biliary secretion is more or less affected, and the urine is scanty and high-coloured. In hepatalgia, on the contrary, these signs are invariably wanting; there may exist, indeed, constant pain and tenderness over the region of the liver, increased to a certain degree by pressure, but manifest exacerbations, even in the worst cases, occur, which are sufficiently indicative of its paroxysmal character. The functions of the organ may proceed uninterruptedly as in its healthy condition. The tongue may be quite clean, or sometimes, in the centre, there may be a gentle creamy fur, and the urine is generally increased in quantity, and is of a lighter colour than ordinary." Treatment of Heputalgia.-_" Gentle purgatives, combined with colchicum, ipecacuanha; and hyoscyamns, will seldom fail to work a speedy cure; and if the constitution have suffered from protracted, unmitigated pain, alkaline vegetable tonics will effect that which we might in vain expect from the rough, insoluble, mineral preparations."-Dr. Allnat, Medicul Gazette.

\section{AN AMERTCAN 'DOCTOR'S SHOP.}

Dr. Drake, in an introductory lecture, at the Louisville Medical School, alluding to the habits of his professional friends, says-

"My firm opinion is, that one of the causes which retard us, in raising our profession, in the West and South, to an excellence which at present seems almost hopeless, is the style in which our offices are fitted up and kept. Who can read and think with method, or sound logic, while everything around him is dirty and disordered; his little stock of furniture displaced, as if a riot had just passed away; his books scattered on chairs, tables, and the greasy medicine shelves; in his book-cases, volumes of different sets mixed together, some lying flat, and some, like the ideas of theis reader, upside down; his skeleton exposed, and joint after joint torn off; his medicines unlabelled, and thrown into a chaos as great as a treatise on the materia medica in the fourteenth century; bundles untied, and bottles left uncorked, or stopped with plugs of paper; dead flies in the ointment within his jars, while others are wading through that which has lain so long spread over his counter, that their feet are blistered by its rancidity; his spatulas foul and rusty; his scales tied with strings and balanced with pieces of paper ; his mortar about as clean as the ancient Kentucky hommony block, which, in the same day, contained the food if the family, and of the family cow and horse, as it stood convenient to all the parties, on nentral ground, near the door of the cabin; his surgical instruments oxidated and rusting away, like his mind; his study table, covered with loose papers and inedical journals (even the Western) with their covers torn off; his walls overspread with a tapestry of cobwebs; his windows as opaque from the dust as the painted glass of an ancient cathedral; his foul candlestick standing all day on his lexicon, and his floor spotted over with the blood of his surrical patients, and his own tobacco juice? The human intelleet cannot act when thus encompassed. Ideas will not arrange themselves; nor will their foul surfaces cohere. The scene re-acts upon his mind, and a chios within rivals that without."-Boston Medical and Surgical Journal.

\section{ERECTILE TUMOUR TREATED BY INOCULATION WITH} CRO'TON OIr.

Mr. Une describes, in the Medical Gazette, this practice, as being successful in the case of a child on whose eyelid a small erectile tumour, the size of a currant, existed. He says,

" The use of the seton, escharotics, or vaccination, would have been equally contraindicated, owing to the risk of violent irritation, or of sloughing. The sole object was to create inflammation enough in the parenchyma of the nævus, and which might be perfectly under control. For the plan of treatment by inoculation with croton oil, the profession is indebted to M. Lafargue, a French surgeon. Not more than five or six punctures ought to be made at one time. A common lancet may be used for the purpose, just as in vaccinating."

The same gentleman says, that in two cases of hæmorrhoids, in which it was necessary to produce a slough, he has successfully applied

CHROMIC ACID AS AN ESCHAROTIC.

" It is a powerful oxidising agent, yielding half its oxygen readily to organic substances, and being reduced to sesquioxide It is exceedingly convenient for application, inasmuch as it consists of a thick crystalline pap, which, when rightly managed, does not spread beyond the prescribed limits; and so soon as its erosive operation is finished, passes into the state of inert pulverulent sesquioxide above mentioned."

In the Boston Medical and Surgical Journal, Dr. DAvis describes a case rarely met with-viz.,

\section{STIRANGULATION OF THE COLON BY AN OPENING IN THI} MESO-COLON.

A merchant suffered from severe paroxysmal pain in the abdomen and spasm of the abdominal muscles, with constipation. After suffering for two or three days, he was reliered by purgatives combined with morphia. Eight months subsequently, he was seized with similar symptoms; obstinate vomiting was now present. Similar treatment was applied, but now unsuccessfully.

"The stomach was found, after death, empty, with considerable ecchymosis; the gall-bladder fully distended with dark bile; the intestimes filled with gas, and a little fluid fæcal matter. In the hypogastrium appeared a large knuckle of intestine, of a deep port-wine colour, composed of thirteen inches of the colon, strangulated in an aperture in the meso-colon, the apertare being about the size of an American quarter of a dollar."

Dr. Bexurngham, in an essay read before the Surgical Society of Dublin, and published in the Medical Press, arrives at the following conclusions on

THE TREATMENT OF ANEURISM BY COMPRESSION.

" 1 st. That the arteries to which pressure is applicable being far more frequently the subject of spontaneous anewrism than those to which it is inapplicable, compression promises to supersede the ligature in the great majority of cases.

" 2 ndly. Pressure has several obvious advantages over the ligature, being applicable to a considerable number of cases in which the ligature is contraindicated or inadmissible.

" $3 \mathrm{rdly}$. The treatment of aneurism by compression does not involve the slightest risk, and even if it should fail, its employment not only does not preclude the subsequent operation by ligature, but renders the chances of the ligature more favourable.

"4thly. Such an amount of pressure as will cause inflammation and adhesion of the opposed surfaces of the vessel at the point compressed is never necessary.

" "5thly. Compression should not be carried even so far as com. pletely to intercept the circulation in the artery at the point comrressed; the consolidation of the aneurism will be more certainly and more quickly brought about, and with less inconrenience to the patient, hy allowing a feeble current of blood to pass through the sac of the aneurism.

"6thly. Compression by means of two or more instruments, one of which is alternately relaxed, is much more effectual than by any single instrument.

" 7 thly. Compression, according to this method, is neither very tedious nor very painful, and can be maintained, in a great measure, by the patient himself.

" 8thly. An aneurism cured by compression of the artery above the tumour, according to the mode laid down, is much less likely to return than when the ligature had been employed." 